\title{
Random Subnetworks of Random Sorting Networks
}

\author{
Omer Angel \\ Department of Mathematics, University of British Columbia, \\ 121-1984 Mathematics Road, Vancouver, BC V6T 1Z2, Canada. \\ angel at math dot ubc dot ca \\ Alexander E. Holroyd \\ Department of Mathematics, University of British Columbia, \\ 121-1984 Mathematics Road, Vancouver, BC V6T 1Z2, Canada. \\ and \\ Microsoft Research, 1 Microsoft Way, Redmond, WA 98052, USA. \\ holroyd at math dot ubc dot ca
}

Submitted: Nov 19, 2009; Accepted: Apr 4, 2010; Published: Apr 19, 2010

Mathematics Subject Classification: 60C05, 05E10, 68P10

\begin{abstract}
A sorting network is a shortest path from $12 \cdots n$ to $n \cdots 21$ in the Cayley graph of $S_{n}$ generated by nearest-neighbor swaps. For $m \leqslant n$, consider the random $m$ particle sorting network obtained by choosing an $n$-particle sorting network uniformly at random and then observing only the relative order of $m$ particles chosen uniformly at random. We prove that the expected number of swaps in location $j$ in the subnetwork does not depend on $n$, and we provide a formula for it. Our proof is probabilistic, and involves a Pólya urn with non-integer numbers of balls. From the case $m=4$ we obtain a proof of a conjecture of Warrington. Our result is consistent with a conjectural limiting law of the subnetwork as $n \rightarrow \infty$ implied by the great circle conjecture of Angel, Holroyd, Romik and Virág.
\end{abstract}

\section{Introduction}

Let $S_{n}$ be the symmetric group of all permutations $\sigma=(\sigma(1), \ldots, \sigma(n))$ on $\{1, \ldots, n\}$, with composition given by $(\sigma \tau)(i):=\sigma(\tau(i))$. For $1 \leqslant s \leqslant n-1$ denote the adjacent transposition or swap at location $s$ by $\tau_{s}:=(s s+1)=(1,2, \ldots, s+1, s, \ldots, n) \in S_{n}$.

Key words: sorting network, random sorting, reduced word, Polya urn Funded in part by Microsoft Research and NSERC. 


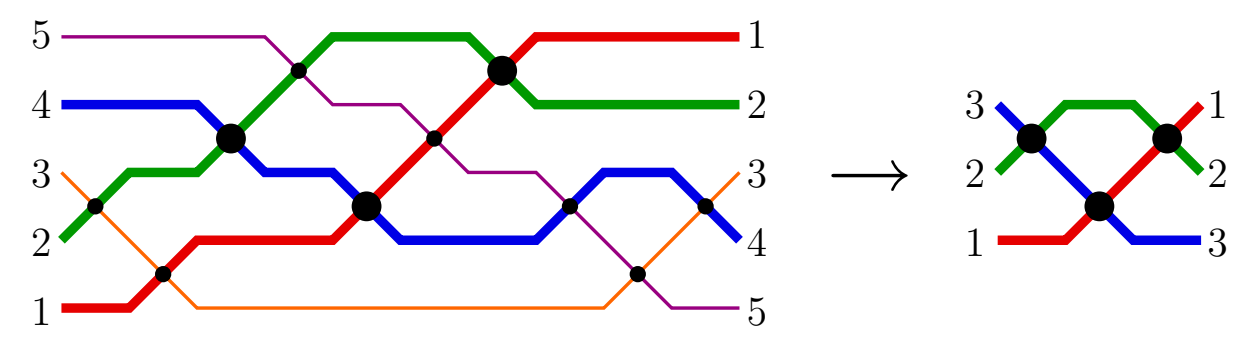

Figure 1: An illustration of the 5-particle sorting network $\omega=(2,1,3,4,2,3,4,2,1,2)$, together with the 3 -particle subnetwork $\left.\omega\right|_{A}=(2,1,2)$ induced by the subset of particles $A=\{1,2,4\}$.

Denote the identity id $:=(1,2, \ldots, n)$ and the reverse permutation $\rho:=(n, \ldots, 2,1)$. An $n$-particle sorting network is a sequence $\omega=\left(s_{1}, \ldots, s_{N}\right)$, where $N:=\left(\begin{array}{l}n \\ 2\end{array}\right)$, such that

$$
\tau_{s_{1}} \tau_{s_{2}} \cdots \tau_{s_{N}}=\rho
$$

For $1 \leqslant t \leqslant N$ we refer to $s_{t}=s_{t}(\omega)$ as the $t$-th swap location, and we call the permutation $\sigma_{t}=\sigma_{t}(\omega):=\tau_{s_{1}} \cdots \tau_{s_{t}}$ the configuration at time $\boldsymbol{t}$. We call $\sigma_{t}^{-1}(i)$ the location of particle $i$ at time $t$.

Given an $n$-particle sorting network $\omega$ and a subset $A$ of $\{1, \ldots, n\}$ of size $m$, the induced subnetwork $\left.\omega\right|_{A}$ is the $m$-particle sorting network obtained by restricting attention to the particles in $A$. More precisely, if the elements of $A$ are $a_{1}<a_{2}<\cdots<a_{m}$, delete from each configuration $\sigma_{t}$ of $\omega$ all elements not in $A$, and replace $a_{i}$ with $i$, to give a permutation in $S_{m}$, then remove all duplicates from the resulting sequence of permutations; the result is the sequence of configurations of $\left.\omega\right|_{A}$. See Figure 1.

The uniform sorting network $\omega_{n}$ is a random sorting network chosen according to the uniform measure on the set all $n$-particle sorting networks. For $m \leqslant n$, the random $\boldsymbol{m}$-out-of- $\boldsymbol{n}$ subnetwork $\omega_{m}^{n}$ is the random $m$-particle sorting network $\left.\left(\omega_{n}\right)\right|_{\mathcal{A}}$, where $\omega_{n}$ is a uniform $n$-particle sorting network, and $\mathcal{A}$ is an independent uniformly random $m$-element subset of $\{1, \ldots, n\}$.

Uniform sorting networks were investigated in [3], leading to many striking results and conjectures. (A different probability measure on sorting networks was considered in [2].) Our main result is the following surprising fact about random subnetworks. We denote the falling factorial $(a)_{r}:=a(a-1) \cdots(a-r+1)$ (so $\left.r !=(r)_{r}\right)$.

Theorem 1. Let $m \leqslant n$. In the random m-out-of-n subnetwork, the expected number of swaps in location $j$ does not depend on $n$, and equals

$$
\mathbb{E} \#\left\{t: s_{t}\left(\omega_{m}^{n}\right)=j\right\}=\frac{\left(j-\frac{1}{2}\right)_{j-1}\left(m-j-\frac{1}{2}\right)_{m-j-1}}{(j-1) !(m-j-1) !}, \quad 1 \leqslant j \leqslant m-1 .
$$

Given only that the left side does not depend on $n$, the formula on the right side may be recovered by reducing to the case $n=m$, which gives $\omega_{m}^{n}=\omega_{m}$, and using known results on the uniform sorting network (specifically, Proposition 5 below). 
It is natural to seek generalizations of Theorem 1. For example one might ask whether the law of $\#\left\{t: s_{t}\left(\omega_{m}^{n}\right)=j\right\}$ is the same for each $n \geqslant m$. This is true for $m=3$ (indeed the law of $\omega_{3}^{n}$ is the same for all $n \geqslant 3$ ), but fails for $m=4$ with $n=4,5$.

From the case $m=4$ of Theorem 1 we deduce the following result, which was conjectured by Warrington [8]. (We abbreviate $\left(s_{1}, \ldots, s_{N}\right)$ to $s_{1} \cdots s_{N}$ ).

Corollary 2. For all $n \geqslant 4$, the probability that the random 4-out-of-n subnetwork lies in $\{123212,321232,212321,232123\}$ is $1 / 4$.

The present work was triggered by Warrington's conjecture. Conjecture 2 has a natural interpretation in terms of geometric sorting networks, which we define next. Consider a set of $n$ points in $\mathbb{R}^{2}$ with no three collinear, no two pairs determining parallel lines, and no pair in the same vertical line. The associated geometric sorting network is defined as follows. Label the points $x_{1}, \ldots, x_{n}$ in order of their projections onto the horizontal axis. For all but finitely many angles $\theta$, the projections onto the line through 0 in direction $\theta$ fall in an order $x_{\sigma(1)}, \ldots, x_{\sigma(n)}$ corresponding to a permutation $\sigma=\sigma_{\theta}$ of the original order, and as $\theta$ is increased from 0 to $\pi$, these permutations form the configurations of a sorting network.

The four networks listed in Corollary 2 are precisely those geometric networks in which one point is in the convex hull of the other three. It turns out that all $n$-particle sorting networks are geometric for $n \leqslant 4$, but not for $n \geqslant 5$, as proved in [6]. In fact it is proved in [1] that the uniform sorting network is non-geometric with probability tending to 1 as $n \rightarrow \infty$; on the other hand, a principal conjecture of [3] is that in a certain sense the uniform sorting network is approximately geometric.

The conjectures in [3] lead to the following precise prediction for the limiting law of the random $m$-out-of- $n$ subnetwork as $n \rightarrow \infty$. We will prove that Theorem 1 is consistent with this conjecture, thus providing the most detailed confirmation to date of the conjectures in [3].

Conjecture 3. Let $X_{1}, \ldots, X_{m}$ be independent identically distributed random points in $\mathbb{R}^{2}$ chosen according to the Archimedes density

$$
\frac{1}{2 \pi \sqrt{1-x^{2}-y^{2}}}
$$

on the disc $x^{2}+y^{2}<1$, and let $\widehat{\omega}_{m}$ be the associated random geometric sorting network. The random m-out-of-n subnetwork $\omega_{m}^{n}$ satisfies the convergence in distribution

$$
\omega_{m}^{n} \stackrel{d}{\rightarrow} \widehat{\omega}_{m} \quad \text { as } n \rightarrow \infty .
$$

Conjecture 3 is implied by [3, Conjecture 3] (and this is implicit in the discussion at the end of [3, Section 1] and [3, proof of Theorem 5]). The conjecture implies that any statistic of the law of $\omega_{m}^{n}$ should converge to the appropriate limit; we establish that this indeed holds for the expected value in Theorem 1.

Proposition 4. Let $\widehat{\omega}_{m}$ be the random geometric sorting network of Conjecture 3. The expected number of swaps in $\widehat{\omega}_{m}$ at location $j$ equals the right side in Theorem 1. 


\section{Proof of main result}

We will use the following key properties of uniform sorting networks.

Proposition 5 ([3]). Consider a uniform n-particle sorting network, and write $N=\left(\begin{array}{l}n \\ 2\end{array}\right)$.

(i) The random sequence of swap locations is stationary. That is, $\left(s_{1}, \ldots, s_{N-1}\right)$ and $\left(s_{2}, \ldots, s_{N}\right)$ are equal in law.

(ii) The probability mass function $p_{n}$ of the first swap location is given by

$$
p_{n}(k)=\mathbb{P}\left(s_{1}=k\right)=\frac{1}{N} \cdot \frac{\left(k-\frac{1}{2}\right)_{k-1}\left(n-k-\frac{1}{2}\right)_{n-k-1}}{(k-1) !(n-k-1) !}, \quad 1 \leqslant k \leqslant n-1 .
$$

Proposition 5 is proved in [3, Theorem 1(i) and Proposition 9]. For the reader's convenience we also summarize the arguments here. Part (i) follows immediately because $\left(s_{1}, \ldots, s_{N}\right) \mapsto\left(s_{2}, \ldots, s_{N}, n-s_{1}\right)$ is a permutation of the set of all $n$-particle sorting networks. Part (ii) requires more technology. By a bijection of Edelman and Greene [4], the location of the first swap is equal in law to the position along the diagonal of the largest entry in a uniformly random standard Young tableau of shape $(n-1, \ldots, 2,1)$. The mass function of the latter may be computed by using the hook formula of Frame, Robinson and Thrall [5] to enumerate Young tableaux with and without a given cell on the diagonal.

The stationarity of the uniform sorting network will play a key role in our proof of Theorem 1. We remark that the random $m$-out-of- $n$ subnetwork $\omega_{m}^{n}$ is not in general stationary; even $\omega_{4}^{5}$ is a counterexample, as noted in [8]. Stationarity apparently also fails for the random geometric sorting network $\widehat{\omega}_{m}$ of Conjecture 3 (according to simulations), and therefore it presumably fails to hold asymptotically for $\omega_{m}^{n}$ as $n \rightarrow \infty$.

Our proof of Theorem 1 will proceed by relating the mass function $p_{n}$ to a Pólya urn process, which is defined as follows. An urn contains black and white balls in some numbers (which for our purposes need not be integers). At each step, one new ball is added to the urn; if the urn currently contains $w$ white and $b$ black balls, the next ball to be added is white with probability $w /(b+w)$, otherwise black.

Lemma 6. Consider a Pólya urn that initially contains $1 \frac{1}{2}$ black and $1 \frac{1}{2}$ white balls.

(i) The random sequence of colors of added balls is exchangeable (i.e. invariant in law under all permutations affecting finitely many elements).

(ii) After $n-2$ balls have been added, the probability that $k-1$ of them are white equals $p_{n}(k)$.

(Property (i) is well known, for arbitrary initial numbers of balls).

Proof. The probability of adding $k-1$ white followed by $n-k-1$ black balls is

$$
\frac{1 \frac{1}{2}}{3} \frac{2 \frac{1}{2}}{4} \cdots \frac{k-\frac{1}{2}}{k+1} \times \frac{1 \frac{1}{2}}{k+2} \frac{2 \frac{1}{2}}{k+3} \cdots \frac{n-k-\frac{1}{2}}{n}=\frac{2\left(k-\frac{1}{2}\right)_{k-1}\left(n-k-\frac{1}{2}\right)_{n-k-1}}{n !} .
$$

Moreover, the probability of adding $k-1$ white and $n-k-1$ black balls in any given order also equals (1), since we obtain the same denominators, and the numerators in a 
different order. This gives the claimed exchangeability. Therefore, the probability that $k-1$ of the first $n-2$ balls are white is (1) multiplied by $\left(\begin{array}{l}n-2 \\ k-1\end{array}\right)$, which equals the right side in Proposition 5 (ii).

Let $h_{m, k}^{n}$ be the mass function of a hypergeometric distribution, i.e., let $h_{m, k}^{n}(i)$ be the probability of obtaining $i$ white balls when $m$ are chosen at random without replacement from an urn containing $n$ balls of which $k$ are white. So

$$
h_{m, k}^{n}(i)=h_{k, m}^{n}(i)=\frac{\left(\begin{array}{c}
k \\
i
\end{array}\right)\left(\begin{array}{c}
n-k \\
m-i
\end{array}\right)}{\left(\begin{array}{c}
n \\
m
\end{array}\right)}
$$

(where as usual we take $\left(\begin{array}{l}a \\ b\end{array}\right)=0$ if $b \notin[0, a]$ ).

Lemma 7. For integers $n, m, j$ satisfying $m \leqslant n$ and $1 \leqslant j \leqslant m-1$,

$$
\sum_{k \in \mathbb{Z}} p_{n}(k) h_{m-2, k-1}^{n-2}(j-1)=p_{m}(j)
$$

In particular the left side does not depend on $n$.

Proof. Consider the Pólya urn of Lemma 6. When $n-2$ balls have been added, suppose $m-2$ balls are chosen at random from these $n-2$. Then the left side is the probability that $j-1$ of those chosen are white. By the exchangeability in (i), this probability remains the same if we condition on the event that the chosen balls are the first $m-2$ to be added to the urn, but then the probability is clearly $p_{m}(j)$ by Lemma 6 (ii).

We remark that a direct computational proof of Lemma 7 is also possible, using induction on $n$.

Proof of Theorem 1. Consider the random $m$-out-of- $n$ subnetwork $\omega_{m}^{n}=\left.\left(\omega_{n}\right)\right|_{\mathcal{A}}$. Let $q(n, m, k, j, t)$ be the probability that the $t$-th swap in the $n$-particle network $\omega_{n}$ occurs in location $k$, and that this swap corresponds to some swap in location $j$ in the $m$-out-of$n$-network. We note first that $q$ is constant in $t$. To check this, consider the configuration $\sigma_{t-1}\left(\omega_{n}\right)=\tau_{s_{1}} \cdots \tau_{s_{t-1}}$ at time $t-1$. Since $\mathcal{A}$ is a uniformly random $m$-element subset of $\{1, \ldots, n\}$ independent of $\omega_{n}$, it follows that $\sigma_{t-1}(\mathcal{A})$ is also a uniform subset and is independent of $s_{t}$ (even though $s_{t}$ is not independent of $\sigma_{t-1}$ ). The claim therefore follows by the stationarity in Proposition 5(i).

On the other hand we have

$$
q(n, m, k, j, 1)=p_{n}(k) h_{m, 2}^{n}(2) h_{m-2, k-1}^{n-2}(j-1),
$$

since given that the first swap in $\omega_{n}$ has location $k$, the event in question occurs if and only if the $m$ chosen elements comprising $\mathcal{A}$ include the pair $k, k+1$, and exactly $j-1$ of $1, \ldots, k-1$. Now the required expectation is $\sum_{k \in \mathbb{Z}} \sum_{t=1}^{N} q(n, m, k, j, t)$. By the above observations, together with Lemma 7 and the fact that $h_{m, 2}^{n}(2)=\left(\begin{array}{c}m \\ 2\end{array}\right) /\left(\begin{array}{c}n \\ 2\end{array}\right)$, this sum equals $\left(\begin{array}{c}m \\ 2\end{array}\right) p_{m}(j)$. 


\section{Proofs of additional results}

Proof of Corollary 2. It is easy to check that of the 16 4-particle sorting networks, the given 4 each have 3 swaps in location 2 , while the remaining 12 each have 2 . But Theorem 1 gives that the expected number of swaps in location 2 is $9 / 4=(1 / 4) 3+(3 / 4) 2$.

Proof of Proposition 4. Let $X_{1}, \ldots, X_{m}$ be i.i.d. with Archimedes density as in Conjecture 3. We start by noting two properties. First, the projection of $X_{1}$ onto any fixed direction has uniform distribution on $[-1,1]$. Second, the signed distance from 0 of the line through $X_{1}$ and $X_{2}$ has semicircle law, i.e. density function $\frac{2}{\pi} \sqrt{1-r^{2}}$ on $[-1,1]$. (See [3, proof of Theorem 5]).

Since each pair of particles swaps somewhere in $\widehat{\omega}_{m}$, it suffices to compute the probability that a given pair, say those corresponding to $X_{1}, X_{2}$, swap in location $j$ (and then multiply by $\left.\left(\begin{array}{c}m \\ 2\end{array}\right)\right)$. This swap occurs when the rotating projection line is perpendicular to the line through $X_{1}$ and $X_{2}$, at which time the projections of $X_{1}$ and $X_{2}$ coincide, at a point $R$ with semicircle law. This swap is at location $j$ precisely if $j-1$ of $X_{3}, \ldots, X_{m}$ are projected to the left (say) of $R$; but the projections of these points are uniform and independent of $R$. Thus the required expectation is

$$
\left(\begin{array}{c}
m \\
2
\end{array}\right) \int_{-1}^{1}\left(\begin{array}{c}
m-2 \\
j-1
\end{array}\right)\left(\frac{1+r}{2}\right)^{j-1}\left(\frac{1-r}{2}\right)^{m-j-1} \frac{2}{\pi} \sqrt{1-r^{2}} d r .
$$

Leaving aside multiplicative constants and applying the change of variable $t=(r+1) / 2$, the integral reduces to a standard Beta integral (see e.g. [7, p. 148]):

$$
\int_{0}^{1} t^{j-\frac{1}{2}}(1-t)^{m-j-\frac{1}{2}} d t=\frac{\Gamma\left(j+\frac{1}{2}\right) \Gamma\left(m-j+\frac{1}{2}\right)}{\Gamma(m+1)} .
$$

Using $\Gamma\left(\frac{1}{2}\right)=\sqrt{ } \pi$, a routine computation then shows that (2) equals the right side in Theorem 1.

We remark that the last computation may be viewed as an asymptotic version of Lemma 7 in the limit $n \rightarrow \infty$ : the sum in Lemma 7 is a Riemann sum for some integral $\int_{0}^{1} f_{n}(t) d t$, with $f_{n}(t) \rightarrow t^{j-\frac{1}{2}}(1-t)^{m-j-\frac{1}{2}}$.

\section{Open questions}

(i) Does the law of the random $m$-out-of- $n$ sorting network converge as $n \rightarrow \infty$, for fixed $m$ ? (Conjecture 3 makes a specific prediction about the limit, but even its existence is not known.)

(ii) Our use of the Pólya urn can be viewed as a natural way to couple the law of the first swap location $s_{1}\left(\omega_{n}\right)$ for different values of $n$ - indeed the coupling has the property that $s_{1}\left(\omega_{n+1}\right)-s_{1}\left(\omega_{n}\right) \in\{0,1\}$. Is there a natural way to couple the entire uniform sorting networks $\omega_{n}$ and $\omega_{n+1}$ ? In particular, does there exist a joint law of $\omega_{n}, \omega_{n+1}$ and some random set $\mathcal{B} \subset\{1, \ldots, n+1\}$, such that $\omega_{n}=\left.\left(\omega_{n+1}\right)\right|_{\mathcal{B}}$ ? 


\section{References}

[1] O. Angel, V. Gorin, and A. E. Holroyd. A pattern theorem for random sorting networks. In preparation.

[2] O. Angel, A. Holroyd, and D. Romik. The oriented swap process. Ann. Probab., 37(5):1970-1998, 2009.

[3] O. Angel, A. Holroyd, D. Romik, and B. Virág. Random sorting networks. Adv. in Math., 215(2):839-868, 2007.

[4] P. Edelman and C. Greene. Balanced tableaux. Adv. in Math., 63(1):42-99, 1987.

[5] J. S. Frame, G. d. B. Robinson, and R. M. Thrall. The hook graphs of the symmetric groups. Canadian J. Math., 6:316-324, 1954.

[6] J. E. Goodman and R. Pollack. On the combinatorial classification of nondegenerate configurations in the plane. J. Combin. Theory Ser. A, 29(2):220-235, 1980.

[7] P. G. Hoel, S. C. Port, and C. J. Stone. Introduction to probability theory. Houghton Mifflin Co., Boston, Mass., 1971. The Houghton Mifflin Series in Statistics.

[8] G. S. Warrington. A combinatorial version of Sylvester's four-point problem. Adv. in Appl. Math., 2010, arXiv:0910.5945. To appear. 